\title{
GUÍA PARA ELABORAR UNA PROPUESTA DE INVESTIGACIÓN
}

\author{
Ana Isabel Mora Vargas \\ Profesora de la Escuela de Formación Docente \\ de la Universidad de Costa Rica
}

Recibido 30-VIII-2004 • Aceptado 7-IX-2004

\begin{abstract}
Resumen: El presente artículo constituye una guía básica para elaborar una propuesta de investigación, proceso previo a la presentación de un proyecto de investigación. Se pretende ofrecer una herramienta que permita a la población estudiantil del área de educación reconocer la vinculación docencia-investigación y plantear de manera, fácil y precisa sus propuestas de investigación en los cursos que asi lo requieran.

La guía se estructura y organiza en tres capítulos: el problema de investigación, el marco conceptual y el procedimiento metodológico. Cada tema recoge la experiencia acumulada de la autora en la labor docente y de investigación en el nivel de educación superior, se ofrece un fundamento teórico básico y se sugieren actividades de aplicación.
\end{abstract}

Palabras clave: Problema de investigación, marco conceptual, procedimiento metodológico.

\begin{abstract}
This article is a basic guide to elaborate a research proposal, the first step in the process of presenting a research project. The purpose of this guide is to offer education students a tool that will allow them to acknowledge the relationship between teaching and research. As a result, students are able to present their research proposals in a much easier way. The guide is divided into three chapters: the research problem, the theoretical framework, and the methodological procedure. Each chapter summarizes the experience gathered by the author as a both teacher and research in the higher education level. The author also offers some basic theoretical foundations and activities suggested for practical application.
\end{abstract}

Key word: Research problem, theoretical framework, methodology.

\section{Introducción}

El presente documento constituye una guía en construcción para elaborar proyectos de investigación en docencia. Se dirige especialmente al docente en servicio o estudiante en el campo de la educación.

De ninguna manera pretende ser un modelo, por el contrario, ante la pluralidad metodológica en este campo, se constituye en un instrumento didáctico que explica las pautas generales para organizar y estructurar un proyecto de investigación. Se espera que el lector se interese por ampliar cada uno de los temas con bibliografía especializada que debe buscar en diferentes medios escritos o virtuales.

Se puede considerar que el educador es un indagador empírico de su propio quehacer docente, su escenario es el contexto sociocultural del aula, donde se encuentra en contacto directo con la población estudiantil. Esto le permite descubrir o probar sus hipótesis confrontando la teoría con la realidad educativa. No obstante, es necesario desarrollar la habilidad para sistematizar las ideas provenientes de la realidad y confrontarlas con la teoría. De lo contrario, como señala Cazau (2001), se corre el riesgo de "descuidar la organización lógica entre las hipótesis" y, por ende "no se puede organizar el conocimiento en teorías" o a la inversa crear una teoría lógica, pero que no ajusta a la realidad. 
$\mathrm{Al}$ respecto, se pueden diferenciar dos tipos de actividades investigativas, la teórica y la empírica; éstas son diferentes pero complementarias en el ámbito de la investigación científica. Indica Cazau (2001:1), que "la investigación teórica compara ideas entre sí, y la investigación empírica compara ideas con la realidad". Se distinguen dos escenarios: el teórico en la biblioteca y el empírico en el laboratorio. Dado que las dos actividades son complementarias, la diferencia se encuentra en el énfasis que le otorgue la persona que investiga a una de ellas. De tal manera que, algunos docentes-investigadores pueden orientarse hacia la teoría, y otros hacia la experiencia con los hechos de la realidad. En el artículo denominado "Hacia la investigación en docencia: orientaciones básicas" se caracteriza al docente-investigador como la persona, que, en forma natural, reflexiona y valora los hallazgos de su propio quehacer educativo, explicándolos e interpretándolos en relación con la teoría y su experiencia acumulada. La labor del docente generalmente trasciende la explicación y la interpretación, al proponer acciones que contribuyan al mejoramiento de la situación problemática; no obstante, son pocos los que sistematizan los hallazgos y resultados.

En estas pocas páginas se pretende ofrecer una herramienta que permita al estudiantado del área de educación, plantear una propuesta de investigación. Esta constituye un planteamiento teórico y práctico en construcción. Desde esta perspectiva, con la elaboración de una propuesta de investigación se desarrolla un proceso investigativo que permite, al proponente, identificarse y definir claramente qué, cómo y para qué se va a investigar. Durante este proceso, la persona que investiga realiza constantemente los ajustes necesarios y pertinentes antes de ejecutar el proyecto. Son muchos los aspectos que se deben atender antes de que una propuesta se convierta en proyecto de investigación, entre ellos: valorar la secuencia y coherencia entre los elementos de la propuesta así como la pertinencia y viabilidad de la misma.

El contenido del documento se estructura y organiza en tres capítulos: el problema de investigación, el marco conceptual y el procedimiento metodológico, básicos para presentar la propuesta de investigación a evaluación de quien corresponda: docente de curso o alguna comisión acreditada para ese fin.

\section{Capítulo 1: El problema de investigción}

El problema de investigación es el nombre que se le ha dado al primer capítulo del proyecto de investigación; dado que el problema no sólo define el qué, por qué y para qué de la investigación, sino que también enmarca teórica y metodológicamente la propuesta de investigación.

La experiencia con estudiantes que requieren plantear una propuesta de investigación permite apuntar que, por varias razones, el primer capítulo es la parte más difícil: se empieza a conocer un tema, luego a identificarse con una situación problemática, con las posibles variables de estudio, las fuentes bibliográficas relacionadas con el tema y las fuentes de información. Esto conlleva un trabajo de muchas horas, no solo en la búsqueda de información, sino también en el planteamiento, por escrito, del primer capítulo de la propuesta o anteproyecto de investigación.

Este tema se encuentra en las diversas referencias bibliográficas relacionadas con enfoques y metodologías para la investigación; por lo tanto, los aspectos que se consignan en este apartado se elaboran con base en la reflexión teórica de autores como Morán (2002), Barrantes (2000), Hernández (1998), Dobles (1996), Navarro (1994), Kerlinger (1991) y Canales (1986), entre otros.

Para el desarrollo de este apartado se proponen los siguientes objetivos y metas: 


\begin{tabular}{|l|l|}
\hline OBJETIVOS & METAS \\
\hline - $\begin{array}{l}\text { Determinar el tema } \\
\text { de investigación. }\end{array}$ & $\begin{array}{l}\text { Capítulo primero } \\
\text { de la propuesta }\end{array}$ \\
- Identificar y definir el & de investigación \\
problema de investigación & \\
- Plantear los objetivos & \\
\hline
\end{tabular}

Con el propósito de organizar la información se presentan tres apartados: el tema de investigación, la formulación del problema, y los objetivos y metas.

\section{El tema de investigación}

Identificar e identificarse con el tema de estudio, con el problema por investigar y plantear los objetivos y metas del proyecto de investigación es una tarea difícil. En la selección de un tema en el ámbito educativo, se debe reflexionar acerca de aspectos tales como:

- Los intereses, necesidades, prioridades del docente-investigador.

- Las necesidades educativas de la población que se tiene en perspectiva.

- Los antecedentes e importancia del tema.

- La afinidad del tema con la formación académica de quien investiga.

- La relevancia y originalidad del tema en relación con la práctica educativa. Aquí la relación sujetoobjeto de investigación adquiere una dimensión muy particular, el sujeto de investigación es el docente en servicio y el objeto de investigación es la docencia; o sea, lo determina alguna situación específica de su práctica educativa.

- La viabilidad para desarrollar el tema; esto conlleva al análisis de elementos como: fuentes de información, tiempo para realizar el trabajo, costos económicos, recursos didácticos, entre otros.
- La motivación que se tiene con el tema seleccionado.

- Las destrezas de quien investiga para utilizar metodologias específicas, acordes con el sujeto y objeto de estudio.

- La pretensión de generalizar los resultados o de describir e interpretar una situación educativa concreta y emprender acciones de solución o cuál es el alcance de la investigación.

- El impacto educativo y sociocultural del proyecto.

En la determinación del tema de investigación es de gran ayuda realizar:

- El diagnóstico de necesidades educativas, dado que la persona investigadora debe tener una visión global de la situación educativa e identificarse plenamente con el tema. Algunas pautas para elaborar un diagnóstico de necesidades educativas, puede consultarlas en el artículo "Hacia la orientación en docencia; orientaciones básicas", publicado en este mismo número de la Revista Educación.

- Hacer una indagación bibliográfica como punto de partida del proceso investigativo. En este paso se hace una revisión exhaustiva de los trabajos relacionados con el tema en estudio. Revise libros que traten extensamente el tema, artículos de investigación que desarrollen problemáticas similares. Estos últimos son de gran ayuda porque ofrecen información acerca del tratamiento teórico y empírico del problema en estudio, presentan la estructura metodológica que se emplea en la investigación y ofrecen las referencias bibliográficas que pueden ser útiles como fuentes de información.

- Una vez que se tiene claridad en el tema que desea investigar, se sistematiza la información relacionada con el objeto de estudio. Esto permite describir los antecedentes teóricos 
y prácticos del tema y justificar la importancia del mismo.

A partir de ese momento se inicia el proceso para formular el problema de investigación; lo cual conduce al planteamiento del objetivo general, los objetivos específicos y las metas. En este apartado se hace referencia a la identificación y definición del problema de investigación, el cual se presenta en cuatro pasos únicamente con el fin de guiar a quien investiga a ordenar la información; por lo tanto, al elaborar el documento escrito del primer capítulo no es necesario hacer esta subdivisión.

\section{Identificación del problema}

El propósito fundamental del problema de investigación es determinar qué es lo que se quiere investigar (objeto de estudio), por qué es importante realizar esa investigación y para qué se realiza ese estudio; es decir, que se pretende resolver o aportar con ese estudio.

La etapa inicial para identificar un problema se denomina primera caracterización del problema (Canales, 1986). En este momento el docente-investigador tiene mucho camino recorrido; se puede decir que la primera caracterización se inicia con el diagnóstico de necesidades educativas y se va perfilando con la selección del tema de investigación. Sin embargo, todavía el investigador presenta una visión general y vaga de la situación problemática, le pueden surgir dudas e interrogantes, las cuales se van concretando y especificando poco a poco, hasta lograr la identificación del problema a estudiar.

Para la formulación del problema se pueden señalar pasos a seguir. Canales (1986) menciona la identificación del área problema, la delimitación y definición del problema y la formulación del mismo. Este proceso culmina con la definición de los objetivos del proyecto de investigación.
Tomando las ideas de Canales (1986) y las necesidades explicitadas por los estudiantes del área de educación, se sugieren los siguientes pasos para ayudar a la persona que investiga a definir el problema de investigación:

\section{Definición del problema}

Se organiza la información en cuatro puntos: acercamiento al problema de investigación, definición del problema, planteamiento del problema y, planteamiento de objetivos y metas. En el documento escrito no es necesario hacer esta subdivisión, ésta es únicamente con el propósito de que la persona interesada pueda visualizar que para llegar a definir el problema de investigación, se parte de una caracterización general y que cada vez se va logrando un mayor nivel de especificidad de los aspectos posibles a estudiar, hasta llegar a concretar las variables de investigación que le permiten plantear los objetivos y las metas. El problema de investigación se puede enunciar de la manera usual, planteando una interrogante o de manera declarativa, enunciando el propósito de la investigación.

\section{Acercamiento al problema de investigación}

- Planteamiento de interrogantes generales que pueden surgir del tema de investigación.

- Explicación en forma general, desde el ámbito internacional, nacional, regional o comunal, de los esfuerzos que se han realizado (instituciones, organismos, docentes, estudiantes $\mathrm{u}$ otras entidades o personas), para abordar directa o indirectamente las interrogantes generales, planteadas anteriormente. También es importante señalar si esos esfuerzos han tenido implicaciones positivas o negativas. 
- Descripción, desde un ámbito más concreto, de cómo se ha abordado o se podría abordar el tema de investigación.

- Explicación, con base en la información que se ha obtenido del diagnóstico de necesidades educativas, de los aspectos relacionados con el tema de investigación que se podrían estudiar. Es importante señalar varios aspectos (5, 10 o más) que le puedan dar bases al investigador para ir delimitando el tema de investigación.

\section{Definición del problema}

- Depuración del problema, quien investiga debe desechar aquellos problemas triviales, incoherentes y poco útiles, que no compensen el tiempo y los recursos a invertir en la ejecución del estudio.

- Fundamentación teóricamente de los aspectos ya seleccionandos de los puntos anteriores. También puede sustentarse con consultas a autoridades educativas o con la experiencia docente de quien investiga; estas últimas deben ser razones lógicas, concretas y razonables.

- Determinación de los puntos concretos que surgen del análisis anterior y con los que el docente-investigador se identifique plenamente y que sean factibles de ser estudiados. Esto es seleccionar las variables o variables principales o elementos más importantes para el estudio del problema y determinar las relaciones entre las variables seleccionadas.

- Justificación, en términos generales, la necesidad y la importancia de estudiar los aspectos o variables derivadas del punto anterior. Recuerde que detrás de un problema hay una necesidad, por lo tanto, una forma de justificar el estudio es mencionando algunas de las necesidades; también puede preguntarse: para qué sirve hacer este estudio, cuál es el aporte, a quiénes les podrían servir los resultados del estudio.

Una vez identificado un problema, debe tener presente que éste forma parte de un conjunto dentro de un cuerpo amplio de conocimientos. Si se desea obtener una respuesta o solución al problema global se deben realizar estudios sucesivos de problemas de un mismo cuerpo de conocimiento (Canales:1986).

\section{3. $\quad$ Planteamiento del problema}

En el planteamiento del problema es importante tomar en cuenta varios aspectos (Kerlinger, 1991; Canales, 1986):

- Se toma en cuenta que el problema debe expresar una relación de variables. Entendiendo variable como los elementos, aspectos, características que se pretenden estudiar en una población, conjunto de cosas o personas, como el aula. También se puede estudiar un caso de un estudiante, entre otras.

- Se plantea el problema de investigación en términos de la relación de dos o más variables. El problema de investigación debe presentar la variable principal y las variables relacionadas.

- Se formula el problema de investigación por medio de una pregunta vital, pregunta fuerza, o de manera descriptiva. Lo importante no es cómo se plantee sino la claridad en la relación en la relación de las variables.

- Se clarifica el tipo de variables del estudio. La comprobación y verificación de las variables es indispensable en un estudio cuantitativo, dado que las variables deben tener la condición de ser medibles y observables. En la investigación cualitativa 
la información permite describir e interpretar variables no cuantificables como el comportamiento, la actitud y otras, relacionadas con el ser humano en situación.

- Se expresa el problema de investigación en una dimensión temporal y espacial. Es recomendable indicar el lugar, institución educativa, región, comunidad, etc., donde se va a realizar el estudio. También se debe indicar el período que va a cubrir la investigación. En este sentido, es pertinente dar al problema una formulación lógica, adecuada precisando sus límites y su alcance.

- Se reflexiona. Este último aspecto no se refiere a la presentación escrita del problema sino más bien a la viabilidad o factibilidad de la investigación. En este aspecto lo importante es tener seguridad de poder acceder a las fuentes de datos para el desarrollo del estudio y tomar en cuenta el lugar y espacio para realizar la investigación.

\section{Planteamiento de objetivos $y$ metas}

Después de formular el problema de investigación se plantean los objetivos y las metas. La función de los objetivos es expresar los resultados una vez finalizada la investigación.

\section{Formulación del objetivo general.} Se formula un objetivo general con base en el problema de investigación. Se expresa el propósito general de la investigación, se responde a la pregunta qué se va a investigar y qué se busca con la investigación. Se ha generalizado que el objetivo se debe redactar iniciando con un verbo en infinitivo que se pueda evaluar, verificar, refutar, contrastar o evidenciar, explicar o interpretar en un momento dado. Otro aspecto de tomar en cuenta al elegir el verbo es el dominio o categoría donde se ubica la acción del objetivo; por ejemplo, conocimiento (memoria), comprensión, aplicación, análisis, síntesis y evaluación, según las variables o categorías que orienten el proceso investigativo (Caro, 2001). Una forma de elegir el verbo del objetivo general puede estar relacionada con el tipo de estudio; por ejemplo:

Describir (investigación de tipo descriptiva). Explorar (investigación de tipo exploratoria). Explicar (investigación de tipo explicativa). Establecer relaciones (investigación de tipo correlacional).

Formulación de los objetivos específicos. Se derivan del objetivo general; plantean lo que se pretende lograr de las variables de estudio y su interrelación; expresan los alcances que se persiguen; quien investiga debe preguntarse cómo llegar al objetivo general. Al igual que el objetivo general es usual iniciar su redacción con un verbo en infinitivo. Al respecto, es importante que la persona que investiga tenga presente los niveles de aplicación de los objetivos. En consecuencia, el objetivo general debe plantearse en un nivel superior de aplicación que los objetivos específicos, dado que éstos apoyan el cumplimiento del objetivo general.

Planteamiento de metas. Pueden plantearse una o más metas por cada objetivo específico. Estas representan acciones concretas, productos de la investigación. Por ejemplo, si un objetivo específico plantea la elaboración de una guía metodológica para la enseñanza de investigación en sétimo año, la meta podría referirse a la realización de un taller para divulgar y presentar la guía metodológica a los docentes de sétimo año. Una publicación de la guía o un artículo en una revista podrían ser metas de este objetivo. Es importante que la meta tenga posibilidades de realización, dado que cada meta constituye un subproducto de la investigación y la suma de todas las metas es el producto global del estudio. 
Es indispensable que el docenteinvestigador no pierda de vista la coherencia interna entre el planteamiento del problema, el objetivo general, los objetivos específicos y las metas. El siguiente esquema permite visualizar estos elementos y autoevaluar la coherencia y consistencia entre ellos. Se sugiere mantener este esquema en hoja separada para que se esté consultando constantemente, en el planteamiento de los capítulos 2 y 3 de la propuesta.

Relación entre problema, objetivos y metas

\begin{tabular}{|c|c|}
\hline \multicolumn{2}{|c|}{ Problema de investigación } \\
\hline \multicolumn{2}{|l|}{ Objetivo general } \\
\hline $\begin{array}{l}\text { Objetivos específicos } \\
\text { 1.- } \\
\text { 2.- }\end{array}$ & $\begin{array}{l}\text { Metas de investigación } \\
\text { 1.- } \\
\text { 2.- }\end{array}$ \\
\hline
\end{tabular}

Para concluir este capítulo se señalan las actividades relevantes que se deben realizar para obtener el producto señalado en la meta.

Actividades de aplicación

\begin{tabular}{|l|l|}
\hline Metas & Actividades \\
\hline $\begin{array}{l}\text { Capítulo 1: } \\
\text { El problema } \\
\text { de investigación. }\end{array}$ & $\begin{array}{l}\text { 1. Realizar un } \\
\text { diagnóstico de } \\
\text { necesidades educativas. } \\
\text { 2. Buscar referencias } \\
\text { bibliográficas } \\
\text { relacionadas con el tema } \\
\text { en estudio. } \\
\text { 3. Determinar el } \\
\text { problema, los objetivos y } \\
\text { metas de investigación. } \\
\text { 4laborar el } \\
\text { documento escrito del } \\
\text { primer capítulo de la pro- } \\
\text { puesta de investigación. }\end{array}$ \\
\hline
\end{tabular}

Una vez planteado claramente el problema de investigación, los objetivos o hipótesis y evaluada su factibilidad, se sustenta teóricamente. En este sentido, es necesario preguntarse: ¿Qué es un marco teórico o conceptual? El capítulo 2 corresponde a esta temática.

\section{Capítulo 2: Marco conceptual}

Consiste en analizar y exponer teorías, principios teóricos, leyes, enfoques, investigaciones pertinentes para sustentar el objeto de estudio y sus variables. Existen diferentes definiciones al respecto; según Neupert (Canales, 1986), es la descripción, explicación y análisis, en un plano teórico, del problema de investigación y, de acuerdo con Tamayo y Tamayo (Canales, 1986), es un sistema conceptual integrado por hechos e hipótesis o supuestos teóricos vinculadas entre sí, en relación con la investigación. Para algunos autores no existe diferencia entre marco teórico y marco conceptual, marco referencial o referente conceptual; por el contrario, para otros como Méndez (1998), el marco conceptual se deriva del marco teórico. En este sentido, el marco teórico es "la descripción de los elementos teóricos planteado por uno o por diferentes autores y que permiten al investigador fundamentar su proceso de conocimiento". Elaborar el marco teórico implica la adopción de una teoría o desarrollo de una perspectiva teórica, mientras que el marco conceptual está constituido por los conceptos involucrados en las variables. Estas son definidas y delimitadas por el investigador en relación con su marco teórico (Méndez, 1995:98).

En consecuencia, en este documento se le ha dado el nombre de marco conceptual al segundo capítulo porque aborda conceptos involucrados en los objetivos. La persona que investiga debe determinar si es un marco teórico o marco conceptual lo que corresponde a su estudio.

Para el desarrollo de este tema se plantea el siguiente objetivo y la meta que se debería lograr cuando se elabore el marco conceptual. 


\begin{tabular}{|l|l|}
\hline Objetivos & Metas \\
\hline $\begin{array}{l}\text { Plantear los pasos para } \\
\text { elaborar el marco } \\
\text { conceptual de la propuesta } \\
\text { de investigación. }\end{array}$ & $\begin{array}{l}\text { Capítulo 2: } \\
\text { Marco conceptual }\end{array}$ \\
\hline
\end{tabular}

Desde el capítulo anterior se viene revisando bibliografía, mucha de esa información puede servir para este capítulo; pero no es suficiente. Para este momento se tienen identificadas las variables del estudio y la búsqueda se dirige hacia los temas que señalan las variables. Esta revisión de bibliografía debe hacerse con sentido crítico para obtener únicamente la información relevante para el estudio. Es necesario señalar que el marco conceptual no se acaba con el planteamiento de este capítulo en la propuesta o proyecto de investigación, sino que éste se continúa mejorando durante el proceso de investigación. Para la propuesta de investigación es necesario que este capítulo sea suficientemente explicativo para sustentar teórica y empíricamente las variables de la investigación.

Con el propósito de presentar algunas ideas para elaborar el marco conceptual, se organiza la información entre apartados: el propósito del marco conceptual, variables o hipótesis y pasos para elaborar el marco conceptual.

\section{El propósito del marco conceptual}

Surge la pregunta ¿cuál es el propósito del marco conceptual? Al responder esta pregunta se mencionan varias acciones (Canales, 1986; Barrantes, 2000 y Hernández, 2003):

- Ubica el problema y el resultado de sus análisis dentro de un conjunto de conocimientos existentes y orienta, en general, el proceso de investigación.

- Ayuda a precisar y organizar los elementos incluidos en el problema, de manera que se evidencien acciones concretas.

Orienta el estudio en cuanto a la relación de las variables de investigación.

Amplía los horizontes del estudio y guía al investigador en el análisis e interpretación de la información. Es decir, amplía la descripción y análisis del problema de estudio planteado. Los elementos teóricos extraídos de la revisión bibliográfica, estudios y teorías pertinentes al tema de investigación constituyen la base para la descripción y explicación de las hipótesis o supuestos teóricos.

- $\quad$ Establece hipótesis y deja planteadas otras para estudios posteriores.

- $\quad$ Sistematiza la información bibliográfica en aras de explicar un fenómeno o situación.

- $\quad$ Predice cómo se va a manifestar un fenómeno dadas ciertas condiciones.

- Orienta la organización de datos o hechos significativos para descubrir las relaciones de un problema con las teorías ya existentes. Es decir, que los elementos que integran las hipótesis o supuestos teóricos deben expresar la dinámica de sus relaciones en forma descriptiva o esquemática.

- Integra la investigación teoría con la investigación empírica. Los supuestos o proposiciones teóricas deben tener una base científica que sustente la investigación. De manera que, el problema se vincula con una estructura teórica más amplia $\mathrm{y}$, al mismo tiempo, abre caminos para investigaciones ulteriores.

- Concibe su estructura como un sistema conceptual integrado. Es decir, que todos los conceptos, hechos, hipótesis y supuestos deben relacionarse entre sí y con el problema de investigación. 


\section{Las variables e hipótesis del marco conceptual}

El término variable es muy utilizado en la investigación, también el marco conceptual fundamenta científicamente las variables del estudio. Éstas se definen como los elementos, características o atributos que se desea estudiar en el problema planteado (Tamayo citado por Canales, 1986).

Las variables en el marco conceptual constituyen aquellos elementos principales o básicos a estudiar en el problema, éstos son seleccionados por la persona que investiga. Únicamente se enuncian de manera conceptual, en un nivel de abstracción y generalidad. En las etapas siguientes de procedimiento metodológico esas variables se llevan a un nivel operativo que permite la observación del fenómeno en estudio.

$\mathrm{Al}$ concluir una investigación, el marco conceptual explica no solamente las variables a estudiar sino también la relación entre éstas, por medio de las cuales se plantean respuestas o explicaciones al problema que se estudia o hipótesis teórica.

Las hipótesis en el marco conceptual contribuyen a explicar anticipadamente los fenómenos o hechos (variables), que le permiten al investigador acercarse a la realidad. Una hipótesis relaciona, de manera general o específica, una variable con otra. Canales (1986), define hipótesis como una suposición que permite establecer relaciones entre hechos. El valor de una hipótesis reside en su capacidad para establecer la relación entre los hechos y, explicar por qué se producen.

\section{Pasos para elaborar el marco teórico}

La elaboración del marco conceptual implica la revisión de bibliografía antes y durante el proceso de redacción del mismo. Esto obliga a realizar visitas constantes a la biblioteca y a otras fuentes de información. Se sugieren una serie de actividades o pasos para elaborar el marco conceptual, sustentadas en las propuestas de Canales (1986) y Méndez (1998). La elaboración de este capítulo constituye un proceso de investigación, que distingue cierto orden en el desarrollo de las actividades. Se sugiere lo siguiente:

- Revisión exhaustiva de la bibliografía acerca del tema. Esto incluye una recopilación de lo escrito e investigado acerca del tema, implica selección, lectura y crítica del material. En el proceso de revisión de la bibliografía es necesario identificar los elementos, factores y aspectos pertinentes para fundamentar el problema. En la revisión bibliográfica se pueden distinguir tres tipos de fuentes de información:

- Primario (directas) proporcionan datos (publicados) libros, antologías, revistas (artículos).

- Secundario. Consisten en información procesada o reprocesada. Listado de fuentes primarias. Ejemplos: resúmenes de información de primera mano (cualitativa, generalmente no publicada); por ejemplo, videos, películas, congresos, libros, tesis, etc.

- Terciario. Fuentes de segunda mano. Ej. Documentos que reúnen nombres y títulos de revistas, boletines, simposios, documentos de empresas, entre otros.

Elaboración de una síntesis conceptual de las teorías, antecedentes e investigaciones previas acerca del problema formulado. De la revisión bibliográfica se extraen resultados de diferentes teorías, investigaciones y datos estadísticos, que a criterio de quien investiga estén relacionados con el problema. Esto constituye la plataforma para elaborar el marco teórico. Es decir: delimitar el área de 
estudio, concretar los conocimientos existentes acerca del problema.

- Identificación de los aspectos principales a estudiar y las relaciones entre ellos.

- Identificación de los elementos teóricos necesarios para fundamentar el problema. La elaboración de fichas es de gran ayuda en esta etapa de revisión bibliográfica. Estas pueden incluir: una idea, cifras, citas, un resumen, etc.) Las fichas deben incluir todos los datos del texto revisado, por ejemplo si es una cita, indicar además de la bibliografía completa, el número de página y, por supuesto, el texto de la cita, entre comillas si es textual.

- Selección de las variables principales; es decir los elementos más importantes para el estudio del problema. Existe diferentes clasificaciones de variables, la más usual es citarlas como variables dependientes e independientes. Canales (1986) las ha clasificado en variables centrales o principales y secundarias del tema en estudio. La variable central se refiere básicamente al problema (variable dependiente); es decir, las variables que se intentan explicar en función de otras variables. Las variables secundarias son aquellas que ayudan a explicar y analizar el problema (variable independiente); o sea, los factores susceptibles de explicar las variables centrales.

- Descripción de las variables y la relación entre ellas y postulación de las hipótesis. La hipótesis contienen las suposiciones, proposiciones, explicaciones y respuestas a hechos y fenómenos del problema.

- Esquematización de las relaciones entre variables. Sobre la base de las hipótesis planteadas se procede a construir el esquema de relaciones. Esto ayuda al investigador a tener una visión de conjunto de las relaciones y facilita la elaboración del marco conceptual o marco teórico.
- Organización del material para la elaboración del marco conceptual. Se puede iniciar con la descripción general del problema y los elementos teóricos relativos al mismo. Se incluyen las variables conceptuales explicando ampliamente la relación planteada en las hipótesis. Estas pueden ser redactadas en un estilo expositivo y no de manera esquemática.

Actividades de aplicación

\begin{tabular}{|c|c|}
\hline Metas & Actividades \\
\hline $\begin{array}{l}\text { Capítulo 2: } \\
\text { Marco conceptual }\end{array}$ & $\begin{array}{l}\text { Consultar la bibliografía } \\
\text { relacionada con el tema } \\
\text { de estudio. } \\
\text { - Recopilar la información } \\
\text { en fichas bibliográficas, } \\
\text { por ejemplo. } \\
\text { - Elaborar un índice con } \\
\text { con los temas y subtemas } \\
\text { que se van a desarrollar. } \\
\text { - Elaborar un mapa con- } \\
\text { ceptual que le permita } \\
\text { explicar la relación de los } \\
\text { temas y subtemas del } \\
\text { índice. } \\
\text { Redactar el capítulo } 2 \text { de } \\
\text { la propuesta de investi- } \\
\text { tigación. No es necesario } \\
\text { que sea muy extenso, } \\
\text { únicamente lo suficiente } \\
\text { para sustentar teórica y } \\
\text { empíricamente la investi- } \\
\text { gación. } \\
\text { Revisar que todas las } \\
\text { referencias bibliográficas } \\
\text { se encuentren en la biblio- } \\
\text { grafía general de la } \\
\text { propuesta. }\end{array}$ \\
\hline
\end{tabular}

El proyecto de investigación, como se menciona al inicio, consta de tres capítulos: el problema de investigación, el marco conceptual y el procedimiento metodológico. En este momento se han elaborados los dos primeros; por lo tanto, se tiene claridad en el objeto de estudio, se han planteado los objetivos y un acercamiento al marco conceptual. A continuación se señalan los aspectos relevantes del capítulo correspondiente al procedimiento metodológico. 


\section{Capítulo 3: Procedimiento metodológico}

Los componentes de los capítulos 1 y 2 , constituyen las bases para determinar el tipo de investigación, la metodología y técnicas apropiadas para abordar el estudio.

Algunos autores como Smith (1983), Pérez Gómez (1985) y Shulman (1986) discriminan dos paradigmas en la investigación educativa, el cuantitativo y el cualitativo. También señalan un tercer paradigma que denominan paradigma crítico, éste se puede ubicar dentro de la corriente filosófica cualitativa que adquiere relevancia en los años ochenta.

El paradigma que se adopte va a condicionar los procedimientos de estudio que se sigan en la investigación. Cada paradigma mantiene una concepción diferente de lo que es la investigación, cómo investigar, qué investigar y para qué investigar.

En este apartado se abordan aspectos generales de los paradigmas cualitativo y cuantitativo de la investigación, el tipo de investigación, selección de los informantes, selección de los instrumentos de recolección de la información, análisis de los resultados y cronograma. También se presenta una amplia bibliografía que puede ayudar en el estudio de estos temas.

\begin{tabular}{|l|l|}
\hline Objetivos & Metas \\
\hline $\begin{array}{l}\text { Ofrecer lineamientos } \\
\text { para elaborar el } \\
\text { procedimiento } \\
\text { metodológico de la } \\
\text { propuesta de investigación. }\end{array}$ & $\begin{array}{l}\text { Capítulo 3: } \\
\text { Procedimiento } \\
\text { metodológico }\end{array}$ \\
\hline
\end{tabular}

Dado que este documento es dirigido a personas del área de educación que se inician en proceso de investigación, se considera importante mencionar la relación entre algunos términos utilizados en la investigación, tales como: paradigmas, tipo de investigación, diseño de investigación, método y técnicas de recolección de la información. En la revisión bibliográfica se observan diferentes clasificaciones e incluso el uso indistinto de tipos y método de investigación o diseño y método. A continuación se determina una clasificación sustentada en diferentes: Taylor y Bogdan (1986), Canales (1986), Muñoz (1998), Flores (2001) y Hernández (2003). A respecto, se hace necesario que la persona que investiga se interese por ampliar la información de acuerdo con las particularidades del objeto de estudio planteado.

\section{Paradigmas de investigación}

Se considera conveniente iniciar este tema con la definición de términos de uso común en las propuestas de investigación: paradigma, metodología y método. El paradigma se puede concebir como un conjunto de creencias y actitudes, una visión del mundo compartida por un grupo de científicos que implica explícitamente una metodología determinada. Cada paradigma se fundamenta en ciertos supuestos que se deben comprobar en el contexto de su aplicación. El paradigma se selecciona de acuerdo con los supuestos que se ajusten mejor al fenómeno que se está investigando (Taylor y Bogdan,1986, Canales, 1986).

Según Taylor y Bogdan, (1986), el término metodología designa el modo en que se enfocan los problemas y se buscan las respuestas. Esta palabra está compuesta del vocablo método y el sustantivo griego logos que significa juicio, estudio, se puede definir como la descripción, el análisis y la valoración crítica de los métodos de investigación. El término, método se deriva del griego meta: hacia, a lo largo, y odos que significa camino, por lo que se puede entender como el conjunto de operaciones y actividades que se realizan de una manera sistemática para conocer y actuar sobre la realidad investigada. Dicho de otra forma es el camino que se sigue para tratar de alcanzar los objetivos de una investigación. 
El método se sustenta en principios epistemológicos y; por lo tanto, se distingue una relación con determinado paradigma. No obstante, cualquier método se puede usar en apoyo de uno u otro paradigma.

En las ciencias sociales han prevalecido dos grandes perspectivas teóricas: el positivismo y la fenomenología.

El positivismo, con sus principales exponentes Augusto Compte (1857) y Emile Durkeim (1917) citados por Canales (1986), se fundamenta en el positivismo lógico, busca los hechos o causas de los fenómenos sociales, prestando escasa atención a los estados subjetivos de los individuos. Para el positivismo el único conocimiento aceptable es el científico que obedece a ciertos principios metodológicos derivados del método científico. Durkheim (Canales, 1986) señala que el científico social debe tratar los hechos sociales como si fueran "cosas" que ejercen influencia externa sobre las personas.

El positivismo ha sido la filosofía dominante por muchos siglos, adopta como modelo de investigación el determinado por la ciencia objetiva, se basa en los supuestos del naturalismo o ciencia que investiga los objetos, animales, plantas y otros fenómenos naturales. Desde esta perspectiva, el positivismo presenta algunas limitaciones cuando se estudian los fenómenos del ser humano. Éstos son muy complejos para ser estudiados utilizando únicamente los métodos cuantitativos de investigación.

En las últimas décadas han tomado auge los métodos cualitativos, específicamente la fenomenología interpretativa o hermenéutica, para entender y describir aquellos fenómenos humanos que interesan a los profesionales en educación.

La fenomenología posee una larga historia en la filosofía y la sociología, en los que destacan Hegel (1807), Husserl (1913), Berger y Luckman (1967), Bruyn (1962), entre otros. La fenomenología interpretativa como método de investigación se basa en la filosofía de Heidegger; su objetivo es comprender las habilidades, prácticas y experiencias cotidianas, y articular las similitudes y las diferencias en los significados, compromisos, prácticas, habilidades y experiencias de los seres humanos. En este sentido, es un método que consiste en entender los fenómenos sociales desde la perspectiva del actor; en el caso de educación desde la visión de mundo de los docentes, estudiantes y todos los involucrados en el proceso de enseñanza y aprendizaje. De esa forma, examina el modo en que se experimenta el mundo. La realidad que le interesa al investigador es la que las personas perciben. Busca la comprensión de los hechos mediante métodos cualitativos que le proporcionen un mayor nivel de comprensión personal de los motivos y creencias que están detrás de las acciones de las personas.

Desde esta perspectiva los positivistas y los fenomenológicos abordan diferentes tipos de problemas y buscan diferentes clases de respuestas. La persona que investiga debe justificar la escogencia del paradigma en el marco de una clara posición epistemológica, no es la escogencia de una técnica particular la que determina si el estudio es cuantitativo o cualitativo, sino la correlación entre el paradigma y el método.

\section{Método cuantitativo de investigación}

En el paradigma cuantitativo el planteamiento epistemológico parte de la unidad del método científico, adoptando el modelo hipotético deductivo, tanto para las ciencias sociales como para las ciencias naturales de donde proviene. Este planteamiento implica una visión restringida de la realidad social. Analiza y estudia aquellos fenómenos observables que son susceptibles de medición, análisis matemáticos y control experimental. Aboga por el empleo de estrategias cuantitativas, fundamentadas en el método científico.

El método científico considera las siguientes etapas (Bunge, 1983): 
- Planteamiento del problema: reconocimiento de los hechos, descubrimiento del problema y formulación del problema.

- Construcción de un modelo teórico: selección de los factores teóricos relativos al problema, formulación de las hipótesis centrales y suposiciones complementarias y elaboración del esquema de relaciones.

- Deducción de consecuencias particulares. Se buscan teorías y elementos que lo conformen y le den consistencia, y que conduzcan al investigador a deducir los juicios y razonamientos pertinentes. Estas pueden ser: soportes racionales, ya probadas en otros estudios y soportes empíricos, no verificados.

- Prueba de las hipótesis. Una vez definido el marco teórico de la investigación, se procede a determinar la manera en que serán sometidas a prueba las relaciones. Se pueden mencionar dos tipos: entre los hechos (hipótesis) y entre los fenómenos o aspectos (variables). La información obtenida debe ser elaborada o procesada, presentada analizada e interpretada en función del problema planteado.

- Introducción de las conclusiones en la teoría. Se pretende además de encontrar las respuestas a los problemas, lograr que las hipótesis o los postulados teóricos se incorporen a los conocimientos existentes sobre el área.

El rigor y la credibilidad científica se basan en la validez interna y externa y en la confiabilidad. Este criterio científico se refiere a la capacidad del instrumento de producir medidas adecuadas y precisas y de permitir conclusiones correctas, así como a la capacidad de los hallazgos de ser aplicables a grupos similares, pero no bajo estudio. En el empleo tradicional del concepto validez, se utiliza el término de validez interna y validez externa. Es decir, la investigación es fiable, sus datos son sólidos y repetibles.

La validez interna se refiere a la precisión de los datos y si las conclusiones reflejan la realidad empírica. Para lograr medidas claras y precisas se identifican varios indicadores y se justifica su relación con el concepto que intenta medir. Cuando se trata de asuntos controversiales o donde el encuestado puede mentir, se desarrollan varias preguntas que recogen información complementaria sobre los mismos asuntos. Para obtener conclusiones válidas se hace una distinción entre las variables causas (independientes) y las variables efectos (dependientes). El objetivo es tratar de establecer la relación menos ambigua posible entre efecto y causa y para ello se utilizan manipulaciones estadísticas o de diseño. Este enfoque enfatiza las relaciones positivas; es decir, aquellas que ocurren a menudo y por lo tanto caen en errores de omisión.

La validez externa indica que el fenómeno observado no solo se da en el grupo observado sino que es generalizable a grupos similares. Este enfoque basa su poder de generalización en la selección de una muestra escogida al azar con representatividad basada en las variables que el investigador considera importantes. Se considera que los hallazgos son generalizables si se encuentra una probabilidad estadística de que los hallazgos no ocurrieron por accidente. Esto se define estadísticamente del $5 \%$ o $10 \%$ de aceptar como verdad un dato falso.

La confiabilidad se entiende como la capacidad del instrumento, que se está aplicando, de producir medidas constantes acerca de un mismo fenómeno en estudio. Se distingue entre confiabilidad externa e interna. La confiabilidad externa indica que otros investigadores usando los mismos instrumentos pueden observar los mismos hechos. La confiabilidad interna indica que otros investigadores pueden hacer la misma conexión entre las 
definiciones planteadas en la investigación y los datos que se recojan; es decir, pueden elaborar el mismo constructo con base en los mismos hechos.

Los procedimientos metodológicos más utilizados en este tipo de estudios son: el control experimental, la observación sistemática del comportamiento y la correlación de las variables.

Este paradigma de investigación adopta la generalización de los procesos para la explicación de los fenómenos y situaciones estudiadas.

\section{Método cualitativo de investigación}

El paradigma cualitativo de investigación es denominado: naturalista, fenomenológico y más tarde hermenéutico. Constituye una reacción a la hegemonía que mantuvo, durante mucho tiempo, el paradigma positivista especialmente en el campo de las ciencias sociales (Dobles, Zúñiga y García, 1996). En el ámbito socioeducativo, especialmente en el quehacer del docente en el aula, se presentan diferentes problemáticas del ser humano que no se pueden explicar y comprender en toda su magnitud, desde una metodología cuantitativa.

El enfoque cualitativo es holístico, integral, trata de comprender los motivos que generan las reacciones humanas, en su propio contexto.

Dentro de sus características relevantes se pueden señalar las siguientes, fundamentadas en las ideas de: Guba, 1985; Pérez Gómez, 1985; Reichard, 1986; Taylor y Bogdan, 1986; Bizquera, 1989; Pérez Serrano, 1990, entre otros. Se invita al lector a ampliar este fundamento con otras referencias bibliográficas.

- La investigación cualitativa es una estrategia de investigación que se basa en una rigurosa descripción contextual de los hechos o situaciones. Esta descripción debe garantizar la máxima intersubjetividad en la captación de una realidad compleja. Por lo tanto, los datos que son susceptibles de categorización se deben recoger en forma sistemática.

- El propósito de la investigación se debe tener muy claro, dado que orienta la percepción de la realidad y la formulación del problema o situación a investigar.

- La intencionalidad y el significado de la actuación son fundamentales para conocer la realidad social y cultural del ser humano. Es decir, ¿por qué el ser humano actúa de una determinada manera y no de otra?

- La insistencia en la relevancia del hecho o fenómeno, frente al rigor (validez interna) del enfoque racionalista. Es decir, la realidad debe comprenderse dentro de un contexto dado, la realidad es holística, global, polifacética, tangible e intangible, dinámica y en continuo cambio. Dado que la realidad no es estática se hace difícil la división de variables dependientes e independientes como en el enfoque racionalista.

- $\quad$ Se tiene muy en cuenta la realidad social que rodea al individuo, así como la flexibilidad y movilidad inherentes a su personalidad. No existe una única realidad, sino múltiples realidades interrelacionadas (Cuba, 1980 y Pérez, 1990). Se interesa por comprender la conducta humana desde el propio marco de referencia de quien actúa.

Se valora al ser humano por su capacidad y respuesta a determinadas situaciones. En este sentido, es necesario adecuar el método a las características de los individuos que se van a investigar, con el propósito de respetar las características de la persona como tal.

- Aboga por la pluralidad metodológica con el propósito de dar una mejor 
respuesta a la situación existente. De esta manera, se puede acercar a la comprensión racional de la realidad, mediante la adopción de estrategias de investigación específica, singular y propia de la acción humana en el ámbito sociocultural.

- Metodológicamente este tipo de investigación adapta y se acomoda a sus planteamientos epistemológicos. Como estrategias metodológicas se apoya en la investigación participativa, el estudio de casos, la etnográfica y la investigación-acción. Las técnicas más usadas son: la observación participativa, la entrevista, el estudio de casos, el análisis de contenido, cuestionarios abiertos, los grupos de discusión, la etnográfica, entre otras.

- La relación sujeto-objeto es de interacción, especialmente cuando el sujeto es la persona humana. En este tipo de investigación existe una interrelación entre el investigador (a) y el objeto de estudio, hasta el punto que se influyen, mientras que en el paradigma racionalista existe independencia entre el investigador y el objeto.

- En el aspecto axiológico los valores inciden en la investigación. Estos forman parte de la realidad. La investigación es influida por los valores del contexto social y cultural.

- La investigación cualitativa no busca la generalización sino que es ideográfica y se caracteriza por estudiar una situación concreta.

- $\quad$ Es ideográfica por que se describen los fenómenos mediante categorías y subcategorías. Es decir, se coloca la realidad en categorías con el propósito de conseguir una coherencia lógica en el suceder de los hechos o de los comportamientos que están necesariamente contextualizados.

- $\quad$ Puede establecer inferencias posibles de acuerdo con los patrones de categorías. En este sentido, se pueden desarrollar hipótesis individuales que se dan en casos individuales.

- No busca la explicación o la causalidad sino la comprensión del fenómeno. Pretende ofrecer profundidad y a la vez detalle, mediante una descripción y registro cuidadoso.

- Rasgos que definen la metodología cualitativa, según Taylor y Bogdan (1986) y Pérez (1990):

- Es inductiva. El diseño de investigación es flexible. Los estudios se inician con interrogantes vagamente formuladas.

- En la metodología cualitativa el investigador ve el escenario y a las personas en una perspectiva holística, considerados como un todo.

- Se estudia a las personas en el contexto de su pasado y de las situaciones en las que se encuentra.

- Los investigadores son sensibles a los efectos que ellos mismos causan sobre las personas objeto de estudio. El investigador interactúa con los informantes de un modo natural y no obligante.

- Los investigadores tratan de comprender a las personas dentro del marco de referencia de ellas mismas. En este caso es esencial experimentar la realidad tal como otros la experimentan.

- La persona que investiga trata de suspender y apartar sus propias creencias, perspectivas y predisposiciones. Observa las cosas como si estas estuvieran ocurriendo por primera vez.

- Los métodos de investigación cualitativa son humanistas. Es decir, son métodos sobre el modo cómo se ven las personas. Si se estudian las personas cualitativamente se llega a conocerlas en lo personal y a experimentar lo 
que ellas sienten en sus luchas cotidianas.

- Quien investiga da énfasis a la validez de su investigación. Los métodos permiten permanecer próximos al mundo empírico. Estos permiten asegurar un ajuste entre los datos y lo que la gente realmente dice $\mathrm{y}$ hace. A través del proceso de triangulación se llega a contrastar y validar la información obtenida mediante diversas fuentes. Este tipo de metodología permite extraer datos de la realidad social con el fin de ser contrastados desde el prisma del método (información documental y oral, por ejemplo). También se pueden realizar exámenes cruzados de los datos obtenidos, recabar información mediante fuentes diversas de modo que se puedan establecer procesos de exploración en espiral.

- Todos los escenarios y personas son dignos de estudio. Todos los aspectos de la vida social pueden ser estudiados.

- La investigación cualitativa es un arte. Los métodos cualitativos no han sido tan refinados y estandarizados como en el enfoque racionalista. El investigador es un artífice, es alentado a crear su propio método. Se siguen lineamientos orientadores pero no reglas; por lo tanto los métodos sirven a la persona que investiga.

No obstante las diferencias entre los dos paradigmas de investigación, es muy difícil encontrar estudios puros, en un solo paradigma. La persona que investiga puede enmarcar su estudio en alguno de los dos, pero eso no lo limita a considerar el tratamiento de algunas variables con técnicas del otro paradigma. Hernández (2003:21) men- ciona el modelo mixto, el cual "representa el más alto grado de integración o combinación entre los enfoques cualitativo y cuantitativo". Según el autor requieren de una mentalidad abierta y un buen conocimiento y manejo de ambos enfoques. Guba, 1985 y Reichardt; señalan además del cuantitativo y cualitativo, el paradigma crítico-reflexivo y otro emergente que está en construcción; este se presenta como el paradigma del cambio.

\section{El tipo de investigación}

De acuerdo con el alcance de la investigación se puede determinar el tipo de estudio a realizar. La clasificación más general señala cuatro tipos (Hernández, 2003): exploratorios, descriptivos, correlacionales y explicativos.

Las investigaciones exploratorias, tienen por objeto esencial familiarizar al investigador con un tema que no ha abordado antes, novedoso o escasamente estudiado. En este caso los estudios constituyen el punto de partida para estudios posteriores de mayor profundidad.

Los estudios descriptivos son útiles para analizar cómo es y cómo se manifiesta un fenómeno y sus componentes. Describir a la recolección de datos, en el caso de un estudio cuantitativo se refiere a medir y para los cualitativos, recolectar información. Este tipo de estudio busca "especificar las propiedades, las características y los perfiles importantes de personas, grupos, comunidades o cualquier otro fenómeno que se someta a un análisis" (Hernández, 2003:117).

Las investigaciones correlacionadas pretenden visualizar cómo se relacionan o no se relacionan o vinculan diversos fenómenos entre sí, cómo se comporta una variable conociendo el comportamiento de otra variable relacionada. Busca evaluar la relación que existe entre dos o más conceptos, categorías o el grado de relación entre dos variables. 
Los estudios explicativos "están dirigidos a responder a las causas de los eventos, sucesos y fenómenos físicos o sociales". Pretenden explicar "por qué ocurre un fenómeno y en qué condiciones se da éste, o por qué se relacionan dos o más variables" (Hernández, 2003; 126).

Conviene anotar que los tipos de investigación no siempre se presentan puros; se encuentran estudios que los combinan entre sí y obedecen sistemáticamente a la aplicación de la investigación.

\section{Diseño de investigación}

El diseño consiste en la forma práctica y concreta de responder al problema de investigación y a lo planteado en los objetivos. Hernández (2003:184) define el término diseño como el "plan o estrategia concebida para obtener la información que se desea". Un estudio puede seleccionar uno o más diseños de investigación y aplicarlos.

Existen diferentes clasificaciones de diseños, se mencionan a grandes rasgos los dos diseños que presenta Hernández (2003):

La investigación experimental. Está relacionada con estudios de tipo cuantitativo e incluye, según la clasificación de Campbell y Standley (Hernández 2003:187), preexperimentos, experimentos puros y cuasiexperimentos.

La investigación no experimental. Señala estudios de tipo transeccionales o transversales y longitudinales. Estos diseños se aplican en ambos enfoques: cuantitativo y cualitativo. En el enfoque cualitativo, según Creswell (Hernández 2003:188), la biografía, la fenomenología, la teoría de contraste, la etnografía y los estudios de casos, pueden adoptar uno o mas diseños no experimentales.

\section{Selección de los informantes}

La selección de los informantes depende del paradigma de investigación, si este es cuantitativo la elección de la muestra depende del objeto de estudio y del ámbito de la investigación. Es importante recordar que cuanto mayor sea el grupo de estudio, mayor será la generalidad de los datos o estructura obtenida. El tamaño de la muestra depende de la profundidad del estudio, algunos autores indican que alrededor de un $20 \%$ de la población se puede considerar representativo, si los informantes han sido seleccionados al azar. En una investigación cualitativa el número de informantes no es relevante, puede realizarse un estudio de un caso o estudios con grupos pequeños. En el caso de educación, se han hecho investigaciones en un aula o en varias o en toda la institución. La profundidad del estudio es lo que determina quiénes son los informantes claves y de qué manera se va a obtener la información.

\section{Selección de los instrumentos y procedimientos de aplicación}

Una vez conocido el fenómeno que se va a estudiar, se pueden elegir los instrumentos y los procedimientos para obtener la información. Surge la pregunta ¿cuál es la técnica más apropiada?, la respuesta requiere de reflexión e indagación acerca de técnicas de investigación que estén en concordancia con el paradigma, el tipo de investigación, el método. Las técnicas deben ofrecer la condición de no distorsionar o perturbar la verdadera realidad del fenómeno que se estudia y además que debe ofrecer la posibilidad de realizar las observaciones repetidas veces. Algunos estudios, especialmente cualitativos, requieren de grabar entrevistas, filmar acciones pedagógicas, hacer anotaciones pormenorizadas de las circunstancias y situaciones, obtener fotografías, cotejar situaciones, entre otras actividades.

Los instrumentos de investigación que presentan preguntas cerradas se consideran apropiadas para la investigación cuantitativa, mientras que para la investigación 
cualitativa son más útiles aquellas que permiten la observación directa, el diálogo, la entrevista abierta, las discusiones de grupo. No obstante, la elección de la técnica, la elaboración y aplicación de los instrumentos dependen de la experiencia del investigador en el manejo de las técnicas (Lankshear y Knobel, 2002).

\section{Análisis de los datos}

En este apartado solo se indica la forma como se pretende analizar e interpretar la información. Esta actividad es un proceso continuo de examen de la información a medida que se obtiene, clasificándola, formulando preguntas adicionales, verificándola y desarrollando conclusiones. Para el proyecto de investigación se describe, en términos generales, cómo se pretende analizar, interpretar y presentar la información recopilada para dar respuesta a los objetivos de la investigación.

En la investigación cuantitativa normalmente el investigador presenta la información en cuadros y gráficos estadísticos que señalan relaciones de porcentajes, frecuencias, correlaciones estadísticas entre las variables, por citar algunas formas. En la investigación cualitativa la organización y estructura de los datos es creativa, la persona que investiga puede buscar la forma que más se ajuste al tipo de información que obtuvo. Lo más usual si se hacen entrevistas abiertas o cuestionarios abiertos, es elaborar categorías y subcategorías de información que permiten explicar e interpretar el fenómeno estudiado.

\section{Cronograma}

Finalmente se elabora un cronograma de actividades que permite visualizar en qué tiempo y qué actividades se van a desarrollar. El cronograma es muy útil para que quien investiga organice no solo el tiempo destinado al desarrollo del proyecto sino también los recursos financieros, didácticos, el tiempo posible para recopilar la información, especialmente si los informantes son estudiantes y docentes, que no se encuentran disponibles en todo momento.

El cronograma es un instrumento de trabajo que se puede ajustar según las necesidades que emergen en el desarrollo del estudio. Cualquier forma de organizar la información es válida; no obstante, debe tener por lo menos dos elementos: actividades y fechas. En la columna destinada a las actividades se citan las más relevantes, el orden que se le dé a las mismas depende de las preferencias de quien investiga, generalmente responden a un orden lógico de las acciones; por ejemplo, búsqueda de información, selección de informantes, elaboración de instrumentos, aplicación de instrumentos, etc. En la columna de fechas, es importante tomar en cuenta el tiempo destinado al desarrollo de la propuesta de investigación. Una manera fácil y práctica

Ejemplo de cronograma de actividades

\begin{tabular}{|c|c|c|c|c|c|c|c|c|c|c|}
\hline \multirow[t]{3}{*}{ Actividades } & \multicolumn{10}{|c|}{ Año(s) 2005} \\
\hline & \multicolumn{3}{|c|}{ Agosto } & \multicolumn{5}{|c|}{ Setiembre } & \multicolumn{2}{|c|}{ Octubre } \\
\hline & S.1 & S.2 & S. 3 & S.4 & S.1 & S.2 & S. 3 & S.4 & S.1 & S.2 \\
\hline Búsqueda de información bibliográfica & $\mathrm{X}$ & $\mathrm{X}$ & $\mathrm{X}$ & $\mathrm{X}$ & $\mathrm{X}$ & $\mathrm{X}$ & $\mathrm{X}$ & $\mathrm{X}$ & $\mathrm{X}$ & $\mathrm{X}$ \\
\hline Selección de informantes & & & $\mathrm{X}$ & $\mathrm{X}$ & & & & & & \\
\hline Elaboración y validación de instrumentos & & & & $\mathrm{X}$ & $\mathrm{X}$ & $\mathrm{X}$ & & & & \\
\hline Aplicación de instrumentos & & & & & & & $\mathrm{X}$ & $\mathrm{X}$ & & \\
\hline
\end{tabular}

$\mathrm{S}=$ semana 
Actividades de aplicación

\begin{tabular}{|c|c|}
\hline Meta & Actividades \\
\hline $\begin{array}{l}\text { Capítulo 3: } \\
\text { Procedimiento } \\
\text { metodológico }\end{array}$ & $\begin{array}{l}\text { Determinar: } \\
\text { - El paradigma en } \\
\text { que se enmarca la } \\
\text { investigación. } \\
\text { - El tipo de } \\
\text { investigación. } \\
\text { - Las fuentes de } \\
\text { información. } \\
\text { - Las técnicas y el tipo } \\
\text { de datos que se } \\
\text { pretenden recopilar. } \\
\text { - Describa cómo se } \\
\text { pretende analizar e } \\
\text { interpretar la } \\
\text { información. } \\
\text { Elabore un cronogra- } \\
\text { ma de actividades. } \\
\text { Organice la informa- } \\
\text { ción por escrito. } \\
\text { Revise la coherencia } \\
\text { interna de la propues- } \\
\text { ta: capítulos } 1,2 \text { y } 3 .\end{array}$ \\
\hline
\end{tabular}

es indicar los meses y cada uno de estos subdividirlos por semanas. El estilo para presentarlos también es a criterio de la persona que investiga, algunos prefieren señalar con "x", marcar el espacio con sombra o colocar las fechas dentro del espacio.

Al finalizar este proceso, se tiene una propuesta o anteproyecto de investigación lista para ser presentada y defendida ante la persona que lo va a revisar o asesorar en el proceso investigativo. Una propuesta siempre está susceptible a ajustes, conforme el estudiantado va madurando el tema y revisando más bibliografía surgen nuevas inquietudes que le permiten hacer mejoras en el documento. En las sugerencias que se ofrecen en este artículo se rescata la importancia de seguir un orden en la ejecución de las acciones y una revisión constante de la coherencia y pertinencia entre todos los elementos que componen la propuesta. La bibliografía existente acerca del tema presentado es extensa, en el artículo se ofrece no solo la que se cita sino otras, con el fin de que la persona interesada pueda buscarlas y consultarlas.

\section{Referencias bibliográficas}

Ander Egg, Ezequiel. (1971). Introducción a las Técnicas de Investigación Social. Ed. Humanitas, Buenos Aires.

Arteaga Herrera, José y Chávez Lazo, Eduardo. (2000). "Integración docente-asistencial-investigativa). Rev. Cubana Educ Med Super 2000: 14 (2), pp. 184-195.

Balluerka, N. (1999). Planificación de la investigación. Salamanca: Amarú.

Barrantes Echavarría, Rodrigo. (2000). Investigación. Un camino al Conocimiento. Un enfoque Cuantitativo y cualitativo. San José, C.R. EUNED.

Bizquera, R. (1989). Métodos de Investigación Educativa. España: Ediciones CEAC.

Bourdieu, P. y otros. (1987). El oficio del sociólogo. México: Siglo Veintiuno, Editores.

Bunge, M. (1983). La investigación científica. Barcelona: Ariel.

Canales, Francisca; Alvarado, Eva y Pineda Elia. (1986). Metodología de la Investigación. México: Editorial Limusa.

CaroVargas, Belarmino.(2001). "Elaboración de proyectos de investigación”, Universidad de San Buenaventura, coordinación general de postgrados especialización en gerencia educativa. Medellín, Colombia. Recuperado el 20 de marzo del 2002). http://www. ciudadfutura.com/mundopc/cursos/ investigacion/invest1.htm

Carr, W. y Kemmis, S. (1988). Teoría crítica de la enseñanza. Madrid: Editorial Martínez Roca. 
Castro, J.A. y Salgado, A. (1989). Técnicas de investigación en las ciencias del comportamiento. (Vols. I y II). Salamanca: Dpto. de Publicaciones de la Universidad Pontificia de Salamanca.

Cazau, Pablo. (2001). "Investigación teórica e investigación empírica”. http://www.galeon.com/pcazau/artmet invteo.htm

Delgado Morera, Elisa. (1998). Elaboración de Proyectos en Centros Infantiles. San José, Costa Rica: EUNED.

Dobles Izaguirre, Ma. Cecilia; Zúñiga Céspedes, Magaly y García Fallas, Jackeline. (1996). Investigación en Educación. San José, CR: EUNED, BID-FOD.

Elliott, John. (1997). La investigaciónacción en educación. Madrid: Ediciones Morata.

Flores Ochoa, Rafael y Tobón Restrepo, Alonso. (2001). Investigación Educativa y Pedagógica. Bogotá, Colombia: Editorial: Mc Graw-Hill.

García Guadilla, C. (1987). Producción y transferencia de paradigmas teóricos en la investigación socioeducativa. Caracas: Fondo Editorial Tropykos.

Goldman, L. (1973). Luckas y Heidegger: Hacia una Filosofía Nueva. Buenos Aires: Amorrortu Editores.

Gómez, Miguel. (1986). Elementos de Estadística Descriptiva. San José, CR. EUNED.

Guba, E. (1985). Criterios de credibilidad en la investigación naturalística en la enseñanza. En J. Gimeno Sacristán y A. Pérez Gómez. "La enseñanza: su teoría y práctica”. Madrid: Akal Editor.
Heidegger M. (1962). Being and time. San Francisco: Harper.

Hernández, Miguel. (2003). Metodología de la investigación. México: Mac Graw-Hill.

Husserl, E. (1986). Ideas relativas a una fenomenología pura y una filosofía fenomenológica. México: Fondo de Cultura Económica.

Kerlinger, F.N. (1981). Investigación del comportamiento: Técnicas y metodología. México: Interamericana (edición original en inglés, 1979).

Lankshear, Collin y Knobel, Michelle. (2002). Problemas asociados con la metodología de la investigación cualitativa. Recuperado el 20 de abril del 2003 de

http://www.cesu.unam.mx/iresie/ revistas/perfiles/perfiles/87-html/87$\underline{02 . h t m}$

León, O.G. y Montero, I. (1997). Diseño de investigaciones. Madrid: Mc GrawHill (2a edición).

López, Ligia y Van Patten, Elia. (1980). Investigación bibliográfica y confección de trabajos escritos. San José, Costa Rica: EUNED.

Méndez, Carlos. 1998. Metodología. Guía para elaborar diseños de investigación ciencias económicas, contables y administrativas. Bogotá, Colombia: Mc Graw-Hill Interamericana, S. A.

Meza Cascante, Luis G. (2005). "Metodología de la investigación: posibilidades de integración". Revista Comunicaciones. San José, Costa Rica Vol. 12, $\mathrm{N}^{\mathrm{o}}$ 1. Recuperado el 10 junio del 2005, de http://www.itcr.ac.cr/revistacomunicacion/Vol.12 num1/metodologia de la_investigacion.htm 
Montero, Marta. (1989). Corrientes, enfoques e influencias de la investigación cualitativa para Latinoamérica. Washington: AICD. Recuperado de internet el 8 de marzo del 2004 de http://www.iacd.aas.org

Navarro, Carlos (1994). "Prontuario para la investigación” (documento inédito).

Pérez Gómez, A. (1985). Paradigmas contemporáneos de investigación didáctica. En J. Gimeno Sacristán y A. Pérez.

Gómez. "La Enseñanza: su teoría y práctica”. Madrid: Akal Editor, pp. 95-138.

Pérez Serrano, María Gloria. (1990). Investigación-Acción. Aplicaciones al campo Social y Educativo. San José, CR: Dykinson.

Reichardt, Ch. y Cook, T. (1986). Hacia una superación del enfrentamiento entre los métodos cualitativos y los cuantitativos en investigación. Madrid: Ediciones Morata, pp. 25-58.

Rockwell, E. (1988). Perspectiva de la investigación cualitativa sobre la práctica docente. DIDAC Número 12, pp. 22-25.

Sabino, Carlos A. (1986). "El proceso de investigación”. Caracas: Panapo.

Shulman, L.S. (1986). Paradigms and research programs in the study of teaching: A contemporary perspective. En M.C. Wittrock (Ed.), "Handbook of Research on Teaching", Macmillan, New York, pp. 3-36.
Smith, L.M. (1987). "Publising Qualitative Research".En:American Educational Reseach Journal, 24.

Spradley, J. (1980). Observación Participante. New York: Rinehart and Winston.

Tapia, María Antonia. (2000). Apuntes para mis alumnos de metodología de la investigación. Santiago http:// www.angelfire.com/emo/tomaustin/ Met/metinacap.htm

Taylor, S.J.y Bogdan, R.(1986). Introducción a los métodos cualitativos de investigación. La búsqueda de significados. Buenos Aires: Editorial Paidós.

Trigo López, Julio. (1996). “Diagnóstico de salud y diagnóstico educativo. Un enfoque integral". Revista Cubana de Salud Pública 2.

Villanueva, P.; Monsell, A. y Cerda, C. (2001). "Líneas emergentes de investigación a partir de las reformas educativas en España en el ámbito de la "Pedagogía de la Diversidad". Departamento M.I.D.E.- Universidad de Valencia.

Wittrock, Merlin C. (1989). La investigación de la enseñanza. Enfoques teorías y métodos (Tomo Y). México: ediciones PAIDOS.

Zubizarreta, Armando. (1969). La Aventura del Trabajo Intelectual, Fondo Educativo Interamericano, Colombia. 
